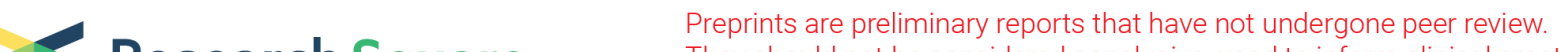 Research Square They should not be considered conclusive, used to inform clinical practice, or referenced by the media as validated information.
}

\section{Achieving Macroscale Liquid Superlubricity Using Lubricant Mixtures of Glycerol and Diols}

haitao yu

Qingdao 971 hospital https://orcid.org/0000-0002-6288-4126

\section{Haigang Pang}

Qingdao 971 Hospital

\section{Gang Yuan}

Qingdao 971 hospital

Dongfang Dong

Qingdao 971 hospital

Ying Lu

Qingdao 971 hospital

Yue Lun

Qingdao 971 hospital

Zhen Li

Qingdao 971 hospital

Qunying Wang

Qingdao 971 Hospital

Wenzhu Dong ( $\square$ wzhdong7@126.com )

Qingdao 971 hospital

\section{Research}

Keywords: Macroscale Liquid, Mixtures of Glycerol, Diols

Posted Date: March 23rd, 2021

DOI: https://doi.org/10.21203/rs.3.rs-348039/v1

License: (c) (1) This work is licensed under a Creative Commons Attribution 4.0 International License.

Read Full License 


\section{Abstract}

\section{Background}

We aimed to assess a serological biopsy using five stomach-specific circulating biomarkers pepsinogen I (PGI), PGII, PGI/II ratio, anti- Helicobacter pylori (H. pylori) antibody, and gastrin-17 (G-17) for identifying high-risk individuals and predicting risk of developing gastric cancer (GC).

\section{Results}

In the cross-sectional analysis, PGII, the PG ratio, G17, anti- $H$. pylori IgG were associated with the presence of $C A G$, and the five biomarkers combined prediction is more effective than single factor prediction $(0.692$ vs $0.54,0.604,0.616,0.629)$.

Conclusion

The combination of pepsinogens, G17 and anti-H. pylori antibodies for serological analysis is helpful to screen CAG high-risk individuals from the general population and recommend that these people should carry out further endoscopy and biopsy.

\section{Introduction}

Gastric cancer (GC) is one of the most common cancers worldwide, and nearly half of new cases and deaths in the world occur in China[1]. Chronic atrophic gastritis (CAG) is an extremely important precancerous in the evolution of gastric cancer[2]. Early diagnosis and monitoring of CAG is of great significance for early detection and treatment of gastric cancer. Currently, endoscopic biopsy histology is the gold standard for the diagnosis of chronic atrophic gastritis[3]. However, gastroscopy use is limited by its invasiveness, high costs and an insufficient supply of skilled endoscopists and endoscopy facilities. Therefore, there is an urgent need for a method to identify CAG in the general individuals.

Recent decades, the examination of gastric biomarkers have been used to diagnose CAG non-invasively, including pepsinogen I (PGI), PGII, the PGI/II ratio, anti- H. pylori antibodies, and gastrin-17 (G-17)[3-8]. Miki[9] established the ABC method, which combines anti- $H$. pylori antibodies with the measurement of serum PG levels, to identify individuals at high risk for future GC development. Tu[10] et al also evaluated a serological biopsy composed of the five stomach-specific circulating biomarkers to stratify individuals' risk of developing GC. However, the practical utility is controversial due to highly varied accuracy in different regions of the world.

Therefore, in order to better understand the relationship between these gastric serological biomarkers and CAG, this study examined the levels of PGI, PGII, the PGI/II ratio, anti- H. pylori antibodies, and G-17 in patients with CAG and chronic nonatrophic gastritis (CNAG). The value of these serum biomarkers in the diagnosis of CAG was preliminarily analyzed to provide further clinical evidence for better prediction rule. 


\section{Materials And Methods}

\section{Study populations}

A total of 186 subjects who came to the 971 hospital for a screening gastroscopy as part of a routine health check-up, were prospectively enrolled in this study from January 2020 to October 2020. The subjects were divided into CAG and CNAG according to endoscopic pathology. Exclusion criteria were a history of $\mathrm{H}$. pylori eradication, previous gastric surgery, treatment with proton pump inhibitors or $\mathrm{H} 2$ blockers within the previous 2 weeks, serious systemic disease, pregnancy, a history of cancer of any type, and those taking anti-secretory or anti-coagulant drugs. A fasting blood sample was obtained from each patient before endoscopy.

\section{Serological measurements and endoscopic and histopathological examinations}

Details on the serological measurements and endoscopic and his[1]topathological examination procedures were previously described (18,19). Serum PGI, PGII, and G-17 concentrations in morning fasting blood samples were measured using enzyme-linked immunosorbent assays (ELISAs; Pepsinogen I ELISA; Pepsinogen II ELISA and Gastrin-17 ELISA kit, Snibe Diagnostic, shenzhen, China), serum anti- $H$. pylori lgG were measured using colloidal gold immunochromatography assay (GICA, anti-H. pylori kit, HUIAN, shenzhen, China).

Gastroscopy with sampling of gastric biopsies was defined as the gold standard for definitive diagnosis. Endoscopic biopsies were obtained from the antrum and corpus, all along the greater curvature (one biopsy from both sites). Biopsy specimens were routinely fixed in neutral formalin and processed in paraffin. Tissue sections were stained with HE, Alcian blue and modified Giemsa (H pylori stain) methods.

\section{Statistical analyses}

SPSS 19.0 was used to carry out statistical analysis. The normal distribution measurement data is expressed as mean \pm standard deviation, the average between the two groups is compared by $t$ test, and the counting data is expressed by rate or percentage, and the comparison is $\chi 2$ test.

H. pylori serological antibody results was classified as negative and positive. The other biomarkers (PGI, PGII, the PGI/II ratio, G-17) were categorized according to quartiles of their distributions in the study cohort. For the cross-sectional analysis, odds ratios (ORs) with $95 \%$ confidence intervals (95\% confidence intervals (Cls)) were calculated using logistic regression.

In the risk prediction modeling analysis, receiver operator characteristic curves with corresponding $\mathrm{C}$ statistics (area under the curve, AUC) based on logistic models were used to measure the discriminatory performance of combination of predictors where the pathology diagnosis was considered the "gold standard". A $P$ value $<0.05$ (two-sided) was considered statistically significant.

\section{Results}


1. Selected baseline characteristics of the study participants

Selected characteristics of the study participants in the cross-sectional analysis are summarized

in Table 1. There was no significant difference in age and sex between the two groups, $(P>0.05)$.

Table 1. Selected baseline characteristics of the study participants

\begin{tabular}{|llll|}
\hline Groups & Cases & Sex(female/male) & Age(years) \\
\hline CAG & 73 & $25 / 48$ & $59.96 \pm 7.87$ \\
\hline CNAG & 113 & $48 / 65$ & $58.17 \pm 6.94$ \\
\hline t/ 22 & & 1.26 & -1.63 \\
\hline P value & & 0.26 & 0.1 \\
\hline
\end{tabular}

2. Cross-sectional associations of gastric biomarkers between CNAG and CAG

As shown in Table 2, the five gastric biomarkers were statistically analyzed between the two groups, in which PGII, the PG ratio, $G 17$, anti- $H$. pylori IgG were statistically significant $(P<0.05)$, while $P G I$ was not statistically significant $(P=0.476)$. There was a dose-response relationship between higher $P G$ II levels or lower PGI/II ratios and the incidence of CAG, and a J-shaped association between G-17 and the incidence of CAG.

Table 2. Cross-sectional associations of gastric biomarkers levels between CNAG and CAG 


\begin{tabular}{|c|c|c|c|c|}
\hline Biomarkers & CNAG(n=113) & $\operatorname{CAG}(n=73)$ & $\mathrm{OR}(95 \% \mathrm{Cl})$ & $P$ value \\
\hline \multicolumn{5}{|l|}{ PGI(ng/ml) } \\
\hline Q1( $\leq 56.97)$ & $29(15.59)$ & 17(9.14) & Reference & 0.476 \\
\hline Q2(56.97-73.22) & $30(16.13)$ & $16(8.60)$ & $0.91(0.388-2.134)$ & \\
\hline Q3(73.22-92.34) & $30(16.13)$ & 17(9.14) & $0.967(0.416-2.248)$ & \\
\hline Q4(>92.34) & $24(12.90)$ & $23(12.37)$ & $1.635(0.714-3.741)$ & \\
\hline \multicolumn{5}{|l|}{ PGII(ng/ml) } \\
\hline $\mathrm{Q} 1(\leq 7.0)$ & $34(18.28)$ & $12(6.45)$ & Reference & 0.028 \\
\hline Q2(7.0-10.52) & $32(17.20)$ & $15(8.06)$ & $1.328(0.54-3.265)$ & \\
\hline Q3(10.52-16.29) & $26(13.98)$ & $21(11.29)$ & $2.288(0.955-5.484)$ & \\
\hline Q4(>16.29) & $21(11.29)$ & $25(13.44)$ & $3.373(1.403-8.11)$ & \\
\hline \multicolumn{5}{|l|}{$\mathrm{PGI} / \mathrm{Il}$ ratio } \\
\hline $\mathrm{Q} 1(\leq 5.345)$ & $24(12.90)$ & $22(11.83)$ & Reference & 0.03 \\
\hline Q2(5.345-7.11) & $23(12.37)$ & $24(12.90)$ & $1.138(0.505-2.568)$ & \\
\hline Q3(7.12-9.11) & $31(16.67)$ & $16(8.60)$ & $0.563(0.244-1.299)$ & \\
\hline Q4(>9.11) & $35(18.82)$ & 11(5.91) & $0.343(0.141-0.836)$ & \\
\hline \multicolumn{5}{|l|}{ G17(pmol/L) } \\
\hline $\mathrm{Q} 1(\leq 2.85)$ & $37(19.89)$ & $9(4.84)$ & Reference & 0.009 \\
\hline Q2(2.85-5.59) & $29(15.59)$ & $18(9.68)$ & $2.552(1.001-6.508)$ & \\
\hline Q3(5.6-12) & $21(11.29)$ & $25(13.44)$ & $4.894(1.929-12.42)$ & \\
\hline Q4(>12) & $26(13.98)$ & $21(11.29)$ & $3.321(1.313-8.4)$ & \\
\hline \multicolumn{5}{|l|}{ Anti-H.pylori IgG } \\
\hline Negative & 74(39.78) & $37(19.89)$ & $1.846(1.012-3.367)$ & 0.045 \\
\hline Positive & $39(20.97)$ & $36(19.35)$ & & \\
\hline
\end{tabular}

3. Receiver-operator characteristic curves of five biomarkers for discriminating of CAG

As shown in Table 2, the PG ratio, G17, anti- $H$. pylori lgG are closely related to the occurrence of $\mathrm{CAG}$, and previous studies have shown that PGI is also an important predictor of CAG[10]. Therefore, we propose a prediction model based on the five biomarkers to identify high-risk populations who may suffer from CAG using the cross-sectional data. The five biomarkers combined yielded a C statistic of 0.692 (95\% 
$\mathrm{Cl}=0.616-0.768)$. Furthermore, combined prediction is more effective than single factor prediction ( 0.692 vs $0.54,0.604,0.616,0.629)$.

Table 3. ROC curves of five biomarkers for discriminating of CAG

\begin{tabular}{|lllll|}
\hline Biomarkers & AUC & Standard error & $95 \% \mathrm{Cl}$ & P value \\
\hline PG囚 & 0.54 & 0.044 & $0.454-0.627$ & 0.354 \\
\hline PG】 & 0.604 & 0.042 & $0.521-0.686$ & 0.017 \\
\hline PGI/II ratio & 0.616 & 0.043 & $0.532-0.7$ & 0.007 \\
\hline G-17 & 0.629 & 0.041 & $0.548-0.71$ & 0.003 \\
\hline Five biomarkers & 0.692 & 0.039 & $0.616-0.768$ & $<0.001$ \\
\hline
\end{tabular}

\section{Discussion}

Atrophic gastritis is a disease in which appropriate glands of the gastric mucosa are lost and replaced by connective tissue and/or intestinal epithelium (intestinal metaplasia)[11]. It is a precancerous lesion of gastric cancer and will evolve into gastric cancer: from chronic active gastritis to atrophy gastritis, to intestinal metaplasia, to dysplasia, and finally to invasive carcinoma[2]. A retrospective study[12] showed that about $6 \%$ of patients with severe gastric mucosal atrophy developed gastric cancer within 5 years. Therefore, it is important to screen out CAG from the general individuals. Gastroscopy combined with histopathological examination is the gold standard for the detection of atrophic gastritis, but the use of endoscopy as a screening test is costly, uncomfortable and does not have good patient's compliance[1314]. Therefore, the emergence of non-invasive serological detection method makes up for the application of this disease screening in the asymptomatic population.

Pepsinogens are products of differentiated gastric mucosa. There are two isoforms of PG (PG I and PG II) present in serum[15]. PGI is produced by the chief cells of the gastric fundus, whereas PG II is secreted by cells of the entire stomach and duodenum[16-17]. Previous studies have shown that, low PG I level and low PG I/II ratio could predict the occurrence of CAG[18], and low PG I/II ratio also is a marker for predicting $G C$, meanwhile elevated PGII levels may be associated with $G C$ and precancerous lesions[10]. In the study, we also found that low PG I/II ratio and elevated PGII levels are CAG risk factors $(P<0.05)$, but there is no clear correlation between $P G I$ and $C A G$ occurrence $(P=0.476)$.

The level of G17 in serum depends on the acidity in the stomach and the number of G cells[19]. Atrophic gastritis in different site may result in variety of G17 concentrations[20]. One study showed that either low or high G17 could leaded to the incidence of gastric cancer[10], and another study showed that individuals with GC had a higher serum level of G-17 than those without[21]. Also some evidences suggested that low G-17 may be related to atrophic gastritis in the stomach antrum, then high serum G-17 may be an indication of atrophic gastritis limited to the stomach fundus/body[22-24]. In our study, we 
divided all the subjects into two groups, and compared with non-atrophic gastritis group, higher G-17 was detected in atrophic gastritis group.

Since the discovery of $H$. pylori, many studies have confirmed that it is associated with gastric cancer and precancerous lesions[25-26]. The present study also showed that $H$. pylori infection was correlated with CAG $(P=0.045)$.

In order to screen gastric cancer and its precancerous lesions more effectively, several studies have tried to establish a variety of methods to identify the high-risk individuals by jointly detecting these serological circulating biomarkers, even commercialized[2,9-10]. However, most of the previous studies are carried out in Europe, the diagnostic reliability of this test remains uncertain[14]. So further evidences are needed to enrich these data in order to establish more accurate prediction rule. In our study, we incorporated the five biomarkers into a prediction model, and the results showed that the combined model could provide more accurate prediction results than the single risk factor model (Table 3). This is similar to the previous studies. The deficiency of this study is that the source of the patients in the single center, and the data is relatively insufficient diversity and representativeness, so that expanded sample size, multi-regional, multi-center clinical studies and further external validation of the usefulness of the prediction model for CAG risk assessment is needed.

In conclusion, our findings support that the combination of pepsinogens, G17 and anti-H. pylori antibodies for serological analysis is helpful to screen CAG high-risk individuals from the general population and recommend that these people should carry out further endoscopy and biopsy. This provides useful data for the establishment of accurate prediction rules for chronic atrophic gastritis.

\section{Declarations}

Competing interests: The authors have declared that no competing interests exist.

Funding: This work was supported by the Qingdao Shinan District Science and Technology Bureau (20202-009-YY). The funders had no role in study design, data collection and analysis, decision to publish, or preparation of the manuscript.

Authors' contributions: Conception and design: Haitao Yu, Qunying Wang, Wenzhu Dong; Statistical support: Haitao Yu, Haigang Pang; Provision of patients: Gang Yuan, Dongfang Dong, Ying Lu, Yue Lun; Collection and assembly of data: Ying Lu, Zhen Li.

Ethics approval: The study protocol was approved by the ethics committees of the respective institutions (971LL-2019012 by No.971 Hospital).

\section{References}


1. Cai Q, Zhu C, Yuan Y, et al. Development and validation of a prediction rule for estimating gastric cancer risk in the Chinese high-risk population: a nationwide multicentre study. Gut. 2019;68(9):15761587.

2. Correa P, Piazuelo MB. Natural history of Helicobacter pylori infection. Dig Liver Dis. 2008;40(7):49049

3. Leung WK, Wu MS, Kakugawa Y, et al. Screening for gastric cancer in Asia: current evidence and practice. Lancet Oncol. 2008;9(3):279-2

4. Malfertheiner P, Megraud F, O'Morain CA, et al. Management of Helicobacter pylori infection--the Maastricht IV/ Florence Consensus Report. Gut. 2012;61(5):646-6

5. Rugge M. Secondary prevention of gastric cancer. Gut. 2007;56(12):1646-164

6. Storskrubb T, Aro P, Ronkainen J, et al. Serum biomarkers provide an accurate method for diagnosis of atrophic gastritis in a general population: The Kalixanda study. Scand J Gastroenterol. 2008;43(12):1448-14

7. Väänänen $\mathrm{H}$, Vauhkonen $\mathrm{M}$, Helske $\mathrm{T}$, et al. Non-endoscopic diagnosis of atrophic gastritis with a blood test. Correlation between gastric histology and serum levels of gastrin-17 and pepsinogen I: a multicentre study. Eur J Gastroenterol Hepatol. 2003;15(8):885-8

8. lijima $K$, Abe Y, Kikuchi R, et al. Serum biomarker tests are useful in delineating between patients with gastric atrophy and normal, healthy stomach. World J Gastroenterol. 2009;15(7):853-85

9. Miki K. Gastric cancer screening by combined assay for serum anti-Helicobacter pylori lgG antibody and serum pepsinogen levels - "ABC method". Proc Jpn Acad Ser B Phys Biol Sci. 2011;87(7):405-4

10. Tu H, Sun L, Dong X, et al. A Serological Biopsy Using Five Stomach-Specific Circulating Biomarkers for Gastric Cancer Risk Assessment: A Multi-Phase Study. Am J Gastroenterol. 2017;112(5):704-715.

11. Dixon MF, Genta RM, Yardley JH, et al. Classification and grading of gastritis. The updated Sydney System. International Workshop on the Histopathology of Gastritis, Houston 1994. Am J Surg Pathol. 1996;20(10):1161-11

12. de Vries AC, van Grieken NC, Looman CW, et al. Gastric cancer risk in patients with premalignant gastric lesions: a nationwide cohort study in the Netherlands. Gastroenterology. 2008;134(4):945-52.

13. Dinis-Ribeiro M, Areia M, de Vries AC, et al; European Society of Gastrointestinal Endoscopy; European Helicobacter Study Group; European Society of Pathology; Sociedade Portuguesa de Endoscopia Digestiva. Management of precancerous conditions and lesions in the stomach (MAPS): guideline from the European Society of Gastrointestinal Endoscopy (ESGE), European Helicobacter Study Group (EHSG), European Society of Pathology (ESP), and the Sociedade Portuguesa de Endoscopia Digestiva (SPED). Endoscopy. 2012;44(1):74-94.

14. Zagari RM, Rabitti S, Greenwood DC, et al. Systematic review with meta-analysis: diagnostic performance of the combination of pepsinogen, gastrin-17 and anti-Helicobacter pylori antibodies serum assays for the diagnosis of atrophic gastritis. Aliment Pharmacol Ther. 2017 Oct;46(7):657667.

15. Samloff IM. Pepsinogens, pepsins, and pepsin inhibitors. Gastroenterology. 1971 Apr;60(4):586-604. 
16. Sano J, Miki K, Ichinose M, et al. In situ localization of pepsinogens I and II mRNA in human gastric mucosa. Acta Pathol Jpn. 1989 Dec;39(12):765-71.

17. Mansour-Ghanaei F, Joukar F, Baghaee $M$, et al. Only serum pepsinogen I and pepsinogen I/II ratio are specific and sensitive biomarkers for screening of gastric cancer. Biomol Concepts. 2019 May 31;10(1):82-90.

18. Lee JY, Kim N, Lee HS, et al. Correlations among endoscopic, histologic and serologic diagnoses for the assessment of atrophic gastritis. J Cancer Prev. 2014 Mar;19(1):47-55.

19. Leung WK, Wu MS, Kakugawa Y, et al. Asia Pacific Working Group on Gastric Cancer. Screening for gastric cancer in Asia: current evidence and practice. Lancet Oncol. 2008 Mar;9(3):279-87.

20. Karimi P, Islami F, Anandasabapathy S, et al. Gastric cancer: descriptive epidemiology, risk factors, screening, and prevention. Cancer Epidemiol Biomarkers Prev. 2014 May;23(5):700-13.

21. Hansen S, Vollset SE, Derakhshan MH, et al. Two distinct aetiologies of cardia cancer; evidence from premorbid serological markers of gastric atrophy and Helicobacter pylori status. Gut. 2007 Jul;56(7):918-25.

22. Sipponen P, Ranta P, Helske T, et al. Serum levels of amidated gastrin- 17 and pepsinogen I in atrophic gastritis: an observational case-control study. Scand J Gastroenterol. 2002 Jul;37(7):785-91.

23. Cao Q, Ran ZH, Xiao SD. Screening of atrophic gastritis and gastric cancer by serum pepsinogen, gastrin-17 and Helicobacter pylori immunoglobulin G antibodies. J Dig Dis. 2007 Feb;8(1):15-22.

24. Sipponen P, Graham DY. Importance of atrophic gastritis in diagnostics and prevention of gastric cancer: application of plasma biomarkers. Scand J Gastroenterol. 2007 Jan;42(1):2-10.

25. Nie Y, Wu K, Yu J, et al. A global burden of gastric cancer: the major impact of China. Expert Rev Gastroenterol Hepatol. 2017 Jul;11(7):651-661.

26. Leung WK, Wu MS, Kakugawa Y, et al; Asia Pacific Working Group on Gastric Cancer. Screening for gastric cancer in Asia: current evidence and practice. Lancet Oncol. 2008 Mar;9(3):279-87.

\section{Figures}




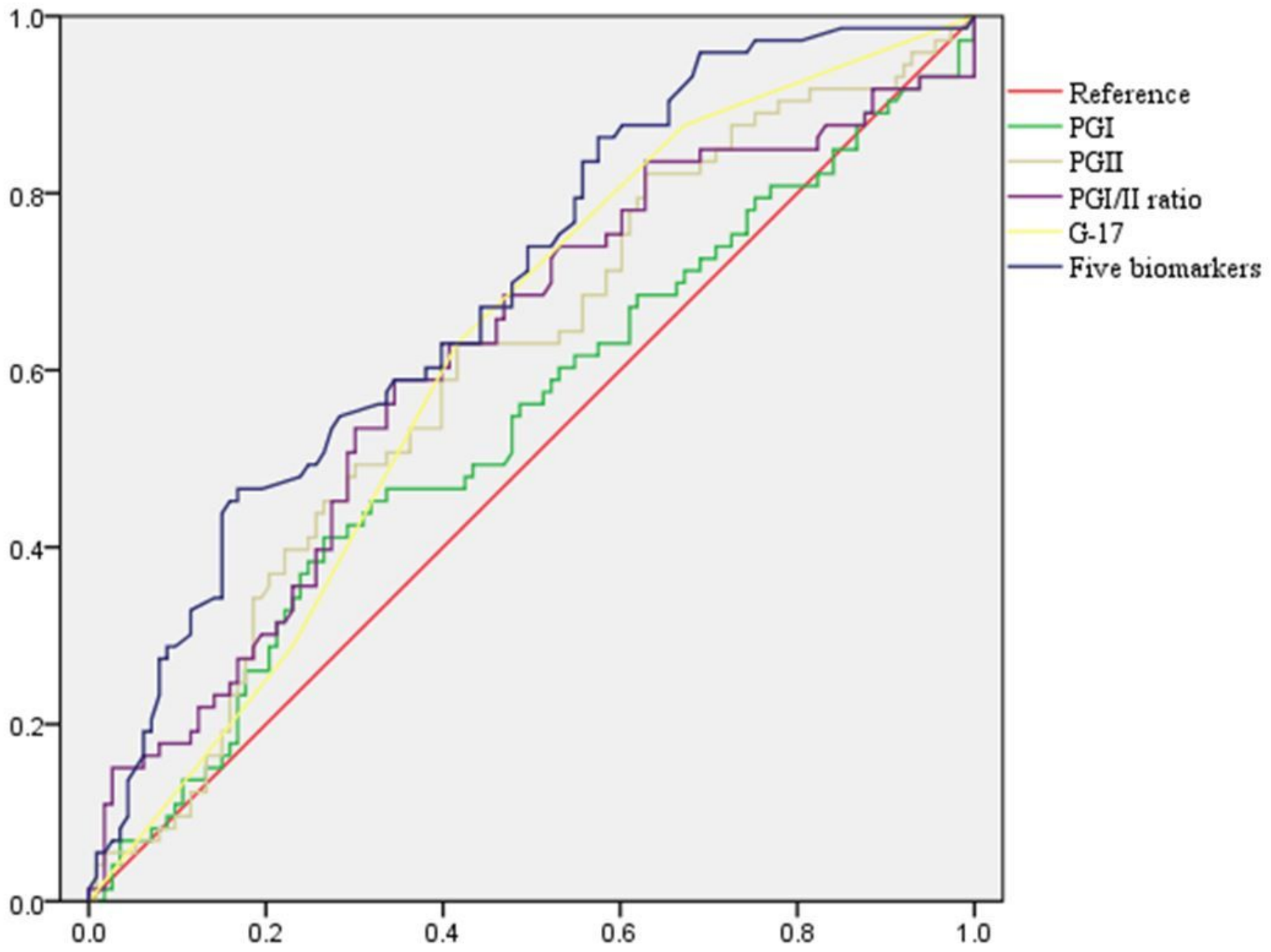

Figure 1

Receiver-operator characteristic curves of PGI, PGII, PGI/II ratio, G-17, and Five biomarkers for chronic atrophic gastritis. 